these needs he formed a minerals utilization branch of the Department. He also found it necessary, partly because of the shortage of paper, to suspend the normal publications of the Department.

Dr. Clegg died unexpectedly after a very short spell of office, so that to Dr. West has fallen the task of the complete reorganization of the Survey in view of the need to recruit a great number of young Indian geologists to provide for a great expansion of departmental activities.

During Oldham's tirne there was no provision in India for geological education, and consequently it was impossible to obtain qualified Indians for the staff. Accordingly, Oldham introduced a system of apprenticeship, but this was not a great success. The first Indian to be appointed after geological education abroad was P. N. Bose (1880), followed by P. N. Dutta (1888); but the Indianization of the Department did not make much progress until the present century, when increasing provision for geological edueation in India, and increased facilities in the form of scholarships for study abroad, ensured a steady supply of well-trained Indian geologists. The position now is that there are only two Europeans left on the graded staff, with a few such officers on short-term agreements for special purposes. The training of the numerous young Indian geologists who have been recruited at a great pace recently has proved a problem, partly solved by sending a batch to the University of Melbourne for training, and partly by the institution of geological survey training camps in India.

The well-known publications of the Department, namely, the Memoirs and the Records of the Geological Survey of India and the Palceontologia Indica, the latter containing descriptions of the fossils, faunas and floras of India contributed by specialists in the various groups all over the world, were all started by Thomas Oldham. There have unfortunately been two breaks in the continuity of the Records, the first during Griesbach's directorship, when he apparently found too troublesome the regular issue of this publication, intended to be quarterly, and instead issued a director's annual report. 'The other break was during the Second World War, when paper was difficult to obtain. An additional journal, entitled Indian Minerals, has been added to the departmental publications since 1947 ; this is issued for the purpose of disseminating information on economic problems and is of a semi-popular nature.

The first edition of the "Manual of the Geology of India", Parts 1 and 2, by Medlicott and Blanford, was, as already mentioned, issued in 1879, and this work was revised in one volume by $R$. D. Oldham (the son of Thomas) in 1893. Sir Edwin Pascoe undertook the preparation of a new edition during his retirement, and a portion of this was in the press and ready for printing off when the Second World War broke out. The paper shortage prevented its issue, and eventually the type-metal, being needed for other purposes, was melted down. After the War the work had to be reset and modificd in view of the number of years that had elapsed. But Sir Edwin Pascoe, beforo his recent untimely death, succeeded in passing the proofs of the first volume, and I undorstand that this has now been issued, though no copies appear yet to have reached (xreat Britain. We may regard as a preliminary to this latest edition of the Manual the latest (fifth) edition of the "Geological Map of India and Adjacent Countries" on the scale of 1 inch to 32 miles, issued in eight sheets in 1928.
Mention must also be made of the most useful of all recent Indian geological publications, T. H. D. La Touche's wonderful "Bibliography of Indian Geology and Physical Geography with an Annotated Index of Minerals of Economic Value", published in four parts during 1917-26.

One other important point requires mention, namely, the changes in the geographical scope of the Geological Survey of India. Up to 1937 the Department was responsible for the geological survey of both India and Burma, but on April 1, 1937, Burma was separated from India, and the new Burma Geological Department was formed, staffed by officers seconded from India. The partition of the Indian Empire on August 15, 1947, necessitated the formation of a Geological Survey of Pakistan, of which Dr. H. Crookshank, then due shortly to retire from the Indian Survey, became the first director, while Dr. W. D. West continued as director of the Geological Survey of India.

Throughout its history the Geological Survey of India has not, however, restricted its scope to the political boundaries of India and Burma. Instead, opportunities have been taken of deputing officers to accompany military and other expeditions, including boundary commissions, so that the Department has contributed largely to our knowledge of the surrounding countries - for example, Blanford in Persia and Abyssinia, Pilgrim in Persia, Mallet in Aden, Griesbach and Hayden in Afghanistan, Stoliczka in Yarkand, Hayden and Heron in Tibet, and Coggin Brown in Yunnan-which explains the words "and Adjacent Countries" in the title of the geological map of India already mentioned.

\section{THE STATISTICAL THEORY OF SOLUTIONS}

A CONFERENCE on "The Statistical Theory of Solutions" was held in the Department of Chemistry, University of Manchester, during the week-end September 30-October 2. The central topic of discussion was the molecular interpretation of free energies of mixing and the other bulk thermodynamic properties of solutions. The interpretation and correlation of these properties is of obvious importance in connexion with phase equilibria, and various speakers showed how statistical theory could be applied to transitions in the solid state, liquid-vapour equilibria in the critical region, and the virial coefficients of mixed gases.

The first meeting, at which Prof. M. G. Evans presided, was opened by Prof. E. A. Guggenheim (Reading). He began with a historical survey of the theory of regular solutions, as developed by Fowler and his school, and went on to describe some new work being carried out at Reading by Mr. M. L. MeGlashan. Leaving aside the question how well the assumptions of the theory describe real solutions, there are two essentially distinct mathematical methods by which it is possible to calculate the free energy of a quasi-crystalline solution. The first method is to expand the free energy of mixing as a power series in $w / k T$, where $w=2 w_{A B}-w_{A A}-w_{B B}$, and $w_{A B}$ is the (free) energy of an $A B$ contect ; this method was later discussed in more detail by Dr. G. S. Rushbrooke. The second is the combinatory method, or its equivalent, the quasi-chemical method. 
No. 4236 January 6, 1951

In this approximation the sites in the pseudo-lattice are considered in pairs, sets of three, or larger groups, and it is assumed that the modes of occupation of these different groups are independent of one another. This assumption will become more accurate the larger the number of sites in a group; and Prof. Guggenheim compared the results of the so-called 'zeroth' approximation with those of the combinatory method applied to pairs, triangles and tetrahedra of sites on a close-packed lattice. The zeroth approximation is surprisingly good, but there should be a definite trend in the calculated free energy as successively larger groups of sites are considered. Mr. McGlashan reported some interesting calculations on the alloy $\mathrm{Cu}_{3} \mathrm{Au}$, where a transition predicted by the zeroth approximation disappears in the first two combinatorial approximations, but reappears when sets of four adjacent sites are considered.

A short account of the method of power series expansion was given by Dr. G. S. Rushbrooke (Oxford). He outlined how it is possible to expand the restrictive grand partition function as a power series in $w / k T$ at high temperatures, or in $\exp (-w / k T)$ at low temperatures. It has not been possible to establish rigorously that either series converges ; but numerical computations made by Mr. A. J. Wakefield for the case of equal activities strongly suggest that the regions of convergence of the two expansions meet at the critical mixing temperature, which is given approximately by $w / k T_{c}=0 \cdot 45$. It seems that, in the absence of an exact solution to the threedimensional lattice problem, the method of power series expansion will probably give the most reliable information available as to the free energy of a quasi-crystalline solution.

Prof. Evans then asked whether it was possible to make any useful statement as to the dependence of the parameter $w$ upon temperature or pressure, since this dependence would have an important effect upon the excess entropy or volume of mixing. In reply, Prof. Guggenheim seid that $w$ is not to be regarded as an ordinary absolute energy; it is defined in terms of the work required to interchange an interior molecule of liquid $A$ with an interior molecule of liquid $B$, and can only be obtained statistically by averaging over all accessible states of the remainder of the system, weighting each state with the appropriate exact Boltzmann factor. For this reason $w$ may be described as a 'co-operative free energy', and would be expected to vary with temperature, though not with composition. Prof. Guggenheim stressed the point that for regular solutions the temperaturedependence of $w$ does not affect calculations of the free energy, which are based on strictly isothermal arguments; but it is not possible to calculate entropies and volumes of mixing without additional information as to how $w$ varies with $T$ and $p$. In the discussion that followed, in which Dr. Rushbrooke and Dr. Longuet-Higgins took part, attention was focused on the difficulty of even writing down the partition function if $w$ is regarded as anything but an absolute energy; allowing $w$ to vary with $T$ makes the theory more elastic, but at the cost of introducing further uncertainties.

At the second session Dr. H. C. Longuet-Higgins (Manchester) described an alternative approach to the theory of solutions. Theories of solution are generally based on some definite or indefinite model of the liquid state, such as the Lennard-Jones cell model or the quasi-crystalline model, and it is difficult to be certain that such a model is statistically consistent with any particular assumptions about the intermolecular forces. However, it is possible to find a set of reasonable assumptions about these forces which enables one to calculate the thermodynamic properties of mixing without appealing to any model at all. It is assumed that, for every pair of molecular species $r$ and $s$ in the solution, the intermolecular energy $u_{r s}$ as a function of distance $\rho$ is given by the equation $u_{r s}(\rho)=f_{r s} u_{00}\left(g_{r s} \rho\right)$, where $u_{00}(\rho)$ is the intermolecular energy function of some chosen com. ponent, and $f_{r s}$ and $g_{r s}$ are constants close to unity. A system satisfying these assumptions may be called a 'conformal solution'; and it can be proved that for a binary conformal solution the excess free energy of mixing at constant $T$ and $p$ is given by the equation

$$
\Delta^{*} G=E_{0}\left(2 f_{12}-f_{11}-f_{22}\right) x_{1} x_{2},
$$

plus terms of the second order in $\left(f_{r s}-1\right)$ and $\left(g_{r s}-1\right)$, where $E_{0}$ is the intermolecular energy of the chosen component, and $x_{1}$ and $x_{8}$ are mole fractions. Now $E_{0}$ is an observable quantity, the variation of which with temperature and pressure can be measured experimentally; so it is possible to correlate the excess free energy, entropy and volume of a conformal solution in terms of the experimental properties of the components; and these predicted correlations are in good agreement with available data on nonpolar solutions. It is interesting to note that, even though the molecular distribution in a conformal solution is random in first approximation, there may nevertheless be a first-order excess entropy of mixing, connected with the temperature variation of $E_{0}$. The succeeding discussion, in which Mr. R. P. Bell (Oxford) and Prof. Guggenheim took part, returned to the interpretation of entropies of mixing ; and it became clear that non-ideal entropies of mixing are not necessarily to be understood in terms of order and disorder.

The next speaker, Mr. D. Cook (Manchester), described the quantitative application of conformal solution theory to phase equilibria in the critical region and, in particular, to the system ethylene carbon dioxide, recently studied experimentally at the Imperial College of Science and Technology, London, by Mr. S. M. Shah and Prof. D. M. Newitt. In order to calculate the $T, p, x$ curves for the twophase region, it is necessary to calculate $E_{0}$ from the equation of state of, say, carbon dioxide; this can be done by extrapolating the available $p, V, T$ data to the requisite low temperatures, but it introduces an unavoidable uncertainty into the theoretical results. Nevertheless, using a value of $2 f_{12}-f_{11}-f_{22}$ derived from the observed critical-temperature curve, one can calculate with considerable accuracy the compositions of phases in equilibrium at a given temperature and pressure; and, in particular, the theory predicts the observed azeotropy in this system, and the direction in which the azeotropic composition varies with temperature.

Mr. McGlashan and Prof. Guggenheim then explained a method of calculating the second virial coefficients of gaseous mixtures from the virial coefficients of the pure components known as functjons of temperature. This method is based on similar assumptions to those of conformal solution theory, except that it is not necessary to assume that the interaction constants $f_{r s}$, etc., are all close to unity. The results agree much more closely with experiment than do those of any previous theory, and details will soon be published elsewhere. It seems, 
therefore, that we are obtaining a better understanding of gas mixtures, and solutions other than those for which the regular assembly is a satisfactory model.

At the third session Prof. I. Prigogine (Brussels) described some recent work extending the LennardJones and Devonshire model of liquids to binary mixtures at temperatures far from the critical point. In such systems it is possible to study the way in which the effective vibration frequency of a molecule in its 'cell' changes with concentration, and also how the mean size of the cells changes with concentration. It is found that both these effects have an influence on the thermodynamic functions, and give important corrections to the usual approximation of regular solutions. The magnitude of these effects has been calculated for the system carbon tetrachloride-carbon tetramethyl, and preliminary measurements give results in good agreement with the theory. Prof. Prigogine also reported some theoretical calculations in which account is taken of the presence in a liquid of Lennard-Jones cells containing 0 or 2 molecules. Such calculations lead to a much better value for the eritical volume of a liquid; but, as yet, the work has not been extended to systems of more than one component. Other topics which arose in the succeoding discussion included the surface tensions of solutions composed of molecules of unequal size or shape; the variation of surface tension with composition gives a sensitive indication of the way in which molecules are oriented in a surface and supplements the information that can be obtained from the bulk thermodynamic properties.

The last session of the conference was devoted principally to the present experimental position. In his introductory paper Prof. D. H. Everett (Dundee) mentioned the scarcity of accurate data on the thermodynamic properties of solutions, and stressed the importance of choosing suitable experimental systems for the testing of particular statistical theories. Older work was concerned primarily with solutions departing little from ideality, but more recently both experimental and theoretical work has been concerned with departures from ideality due to flexibility and differences in shape. Prof. Everett discussed in some detail the system benzene-diphenyl, recently studied with concordant results by several different workers. As he pointed out, the results cannot be quantitatively interpreted in terms of a quasi-crystalline model with benzene occupying one site and diphenyl two; it seems that in dilute solution diphenyl must disorient the benzene structure, and perhaps for this reason the system is not a satisfactory one against which to test the theory. Prof. Everett directed attention to the useful supplementary information that may be derived from measuring non-thermodynamic properties such as viscosity, dielectric constant and absorption spectra. In the critical region very few reliable thermodynamic data are available; and here a few well. chosen measurements should greatly increase our understanding of such phenomena as the solubility of solids in vapours.

In the discussion, Mr. J. H. Baxendale gave a careful analysis of the experimental errors in his investigation with Dr. B. V. Enustin of the benzenediphenyl system, and showed that the discrepancies with the simple theory are well outside the experimental errors. Dr. V. Mathot (Brussels) described a method of calculating the thermodynamic properties of a solution in which the solute consists of monomers and associated complexes. The aetivity coefficient of carbon tetrachloride in mixtures of this liquid with alcohols is found to fall between the value calculated from the Flory-Huggins model and the value appropriate to an 'ideal infinitely associated' solution. Further, it can be shown by the principle of detailed balancing that the ratio of activity coefficients of solute and solvent is equal to the fraction of solute molecules in the monomeric state; and this relationship is verified with great accuracy by spectroscopic measurements on the hydroxyl vibrational absorption band in its first and second overtones. This shows clearly that deviations from ideality are, indeed, due to the formation of complexes in these systems. Another observation which aroused great interest was the fact, reported by Dr. J. H. van der Waals (London), that the heats of mixing of hydrocarbons sometimes vary considerably with temperature; no satisfactory explanation has yet been found for this phenomenon.

Among the visitors who also took part in the conference were Dr. F. Booth (King's College, London), Mr. J. L. Copp and Mr. G. Duff (Dundee), Dr. M. J. S. Dewar and Dr. H. Tompa (Maidenhead), Dr. R. Freeman and Dr. W. Moffitt (Welwyn), Dr. E. L. Mackor (Oxford), Dr. P. Meares (Aberdeen), Dr. G. J. Szasz (American Embassy, London), Mr. Trappeniers (Brussels) and Dr. L. R. G. Treloar (Royal Institution, London).

\section{H. C. LONGUET-HiganNS}

\section{OBITUARIES}

\author{
Dr. L. J. Comrie, F.R.S.
}

Dr. LESLIE JoHN COMRIE died on December 11 at his home in London at the age of fifty-seven. His health had been failing after a stroke two years ago, which left him with an active mind, but with much reduced capacity for work. He worked right up to the last, almost completing a project of long standinga guide to errors in published mathematical tables.

Comrie was born at Pukekohe, New Zealand. $\mathrm{He}_{\mathrm{e}}$ took a degree in chemistry at Auckland University College, before joining the New Zealand Expeditionary Force and serving in the First World War, during which he lost a leg. After the War he gave up chemistry for astronomy and computation, which were to be the great interests of his life. He was elected an Isaac Newton Student at Cambridge, and took his Ph.D. in 1923. He then spent three years in the United States, teaching astronomy and introducing computation into the student courses, first at Swarthmore College, then at North-Western University, Evanston, Illinois.

Returning to England, which he made his home, he served the Nautical Almanac Office first as deputy superintendent, then, during 1930-36, as superintendent. During this period he revolutionized the "Nautical Almanac" and its computation. By mechanizing calculation he greatly increased its range, accuracy and cheapness. This was reflected in great changes in the content of the "Nautical Almanac" itself-the major ones came first in that for 1931-which much increased its value as an astronomical tool.

In 1937 Comrie left the Nautical Almanac Office to found the Scientific Computing Service, Ltd., the first independent professional organization (and still the only one) to provide large-scale computation with high mathematical content. This organization, under 